\title{
Uma Metodologia para Redução do Consumo Energético no Monitoramento Adaptativo de Aplicações em Fog Utilizando Lógica Fuzzy
}

\author{
Julio Machado ${ }^{1}$, André Rauber Du Bois ${ }^{1}$ \\ ${ }^{1}$ Centro de Desenvolvimento Tecnológico - UFPel \\ Pelotas - RS - Brasil \\ \{jmdsneto, dubois\}@inf.ufpel.edu.br
}

\begin{abstract}
Resumo. Com o crescimento de aplicações próximas ao usuário, tornou-se crucial o modo como os dispositivos de borda gerenciam os seus recursos. Aplicações de monitoramento de ambientes precisam lidar tanto com o ambiente sendo monitorado quanto quanto com o gerenciamento de seus próprios recursos. Técnicas foram desenvolvidas buscando lidar com esse desafio, a discutida neste trabalho é o monitoramento adaptativo utilizando propriedades da Lógica Fuzzy para reduzir o consumo energético enquanto mantêm a qualidade do monitoramento.
\end{abstract}

\section{Introdução}

Os Paradigma da Fog Computing e da Internet of Things (IoT) já são uma realidade, com desafios novos em relação ao seu predecessor, o Paradigma da Cloud Computing, que possuem como objetivo permitir o processamento de dados próximo ao usuário, impactando na redução do uso dos recursos de rede, diminuição da latência e a possibilidade da existência harmoniosa de ambientes geo distribuídos [Buyya et al. 2018], permitindo assim um monitoramento de diversos ambientes em tempo real. Entretando, esse monitoramento é realizado de forma limitada, uma vez que os dispositivos de borda não possuem recursos ilimitados.

Este trabalho apresenta uma metodologia para a flexibilização do monitoramento adaptativo utilizando Lógica Fuzzy.

\section{Monitoramento Adaptativo}

Buscando reduzir o volume de dados gerados pelas aplicações de Fog e IoT, é possível adaptar o monitoramento realizado de forma dinâmica para otimizar o uso dos recursos. O monitoramento adaptativo é o processo de ajustar dinamicamente a taxa de amostragem à evolução atual da métrica da aplicação, assim, quando fases estáveis em um fluxo de dados são detectadas, a taxa de monitoramento é reduzida para facilitar o processamento e o consumo de energia. Do mesmo modo, quando os valores desse mesmo fluxo variam em determinado tempo, a taxa de amostragem é aumentada para notificar imediatamente se há alterações críticas no ambiente. Além disso, esse processo permite adaptar dinamicamente a faixa de filtros para acompanhar a evolução da análise sem exigir previamente que os usuários adivinhem qual faixa de filtro deve ser aplicada. Trabalhos como do [Kwon and Noh 2013], [Caglar and Gokhale 2014] e [Stankovski et al. 2016] abordam o monitoramento adaptativo, aliado a técnicas como: limite do uso de recursos, predição 
do uso dos recursos baseado em um histórico como estratégia na redução do consumo energético e o uso de contêiners buscando garantir a Quality of Service (QoS). Entretando, a precisão do monitoramento adaptativo das aplicações é um desafio presente em todos os trabalhos. Visando tornar este monitoramento mais flexível e preciso, este trabalho propõe o uso da Lógica Fuzzy [Zadeh 1988] como estratégia para equilibrar a qualidade do monitoramento com a taxa de coleta dos dados.

\section{Resultados Esperados}

Para o desenvolvimento deste trabalho, foi utilizado o simulador de ambientes e infraestruturas em Fog IfogSim [Gupta et al. 2017] devido a ser o mais utilizado pela comunidade acadêmica.

Para os testes do trabalho, foi utilizado uma aplicação já existente no IfogSim, que simula um ambiente de monitoramento distribuído com câmeras. Desse modo, a abordagem utilizada para flexibilização do consumo energético com a qualidade do monitoramento consiste em equilibrar quantidade de eventos iguais em sequência executados. Seja:

$F_{i}$ o número de eventos iguais em sequência;

$R_{j}$ o número probabilístico de execução dos eventos iguais em sequência;

$V=F_{i}$ AND $R_{j}$ representa a flexibilização entre a qualidade do monitoramento e redução da quantidade de dados processados, por consequência a diminuição do consumo energético.

As duas variáveis, $F$ e $R$, representam a entrada do sitema Fuzzy e $V$ representa a intersecção resultante das entradas. Com isso, poderemos classificar o nível de monitoramento como "baixo", "ideal" ou "excessivo"buscando adaptar o mesmo conforme a evolução das aplicações. A próxima etapa do trabalho visa aplicar esta metodologia a outros tipos de aplicações em Fog no ambiente de simulação IfogSim.

\section{Referências}

Buyya, R., Srirama, S. N., Casale, G., Calheiros, R., Simmhan, Y., Varghese, B., Gelenbe, E., Javadi, B., Vaquero, L. M., Netto, M. A., et al. (2018). A manifesto for future generation cloud computing: research directions for the next decade. ACM computing surveys (CSUR), 51(5):105.

Caglar, F. and Gokhale, A. (2014). ioverbook: intelligent resource-overbooking to support soft real-time applications in the cloud. In 2014 IEEE 7th ICCC, pages 538-545. IEEE.

Gupta, H., Vahid Dastjerdi, A., Ghosh, S. K., and Buyya, R. (2017). ifogsim: A toolkit for modeling and simulation of resource management techniques in the internet of things, edge and fog computing environments. Software: Practice and Experience, 47(9):1275-1296.

Kwon, S.-k. and Noh, J.-h. (2013). Implementation of monitoring system for cloud computing environments. IJMER, 3(4):1916-1918.

Stankovski, V., Trnkoczy, J., Taherizadeh, S., and Cigale, M. (2016). Implementing time-critical functionalities with a distributed adaptive container architecture. In $i i W A S$ 2016, pages 453-457. ACM.

Zadeh, L. A. (1988). Fuzzy logic. Computer, 21(4):83-93. 